\section{Atto pubblico}

Originale No. 1868

Franziska Iseli, notaio del Cantone Berna, con studio a Berna, Bahnhofplatz 3, certifica, che il Segretariato generale dell'associazione Federazione dei medici svizzeri (FMH) con sede a Berna, Elfenstrasse 18, 3006 Berna, Le ha dato l'incarico di formulare un atto pubblico riguardo ai seguenti fatti:

\section{Premesse}

1. Decisione per la votazione generale

La Camera medica svizzera nel corso della sua seduta del 13 dicembre 2001, in base all'art. 30 cpv. 2 let. h e l'art. 23 e 24 cpv. 3 degli Statuti della FMH, ha deciso di mettere in votazione generale tre questioni in merito alla TARMED.

\section{Basi statutarie e regolamenti della FMH}

Secondo la lettera B cifra I del Regolamento d'esecuzione della FMH, approvato dalla Camera medica l'8 aprile 1999, la votazione generale come agli art. 23 e 24 degli Statuti FMH, deve rispettare le seguenti norme (citazione):

"1. Tutti i membri ordinari della FMH hanno il diritto di partecipare alla votazione generale.

2. Le organizzazioni e i membri singoli della FMH possono chiedere una votazione generale su una decisione della Camera medica entro 60 giorni a decorrere dalla data in cui la decisione è pubblicata, simultaneamente nelle tre lingue ufficiali, nel (Bollettino dei medici svizzeri).

3. La votazione generale deve avere luogo entro quattro mesi a partire dalla ricezione dell'ultima domanda valevole o dalla decisione della Camera medica.

4. Il Segretariato generale organizza la votazione generale. La procedura di voto è spiegata nelle tre lingue ufficiali nel «Bollettino dei medici svizzeri`

5. Ogni membro FMH avente diritto di voto riceve in busta chiusa, indirizzata personalmente il necessario materiale di voto, comprendente la scheda di voto, le spiegazioni di voto ed eventuali documenti supplementari. Quest'ultimi possono anche essere allegati o pubblicati nel «Bollettino dei medici svizzeri. La busta contenente il mate- riale di voto inviata ai membri dal Segretariato generale, deve essere conservata e rinviata con la scheda di voto perché vale come documento di legittimazione per il voto.

6. Le schede di voto compilate sono da spedire a un notaio in attività designato dal Segretariato generale. Il notaio verifica che assieme alla scheda di voto ci sia la busta originale di spedizione (controllo del diritto di voto), redige un verbale sulla votazione e comunica i risultati in forma anonima, al Segretariato generale.

7. A parte i due casi citati ai capoversi 4 e 5 dell'articolo 33 degli Statuti FMH, ${ }^{1}$ le decisioni della votazione generale sono prese con la maggioranza assoluta delle schede di voto valevoli rientrate.

8. Il Segretariato generale comunica senza indugio i risultati al Comitato centrale e li pubblica nel ‘Bollettino dei medici svizzeri. Tre mesi dopo la pubblicazione dei risultati, le schede di voto sono eliminate." (Fine della citazione)

\section{Incarico al notaio}

In base alle norme del Regolamento d'esecuzione della FMH, il Segretariato generale della FMH ha incaricato la signora Franziska Iseli, notaio, di eseguire lo spoglio, in forma anonima, della votazione. L'incarico prevede i seguenti compiti:

a) Il notaio deve prendere in consegna le schede di voto compilate.

b) Il notaio deve controllare o far controllare che ci siano le schede di voto e le buste di spedizione della votazione per garantire la legittimità dei voti.

c) Il notaio esegue o fa eseguire il conteggio in forma anonima di tutti i voti a lui arrivati entro il 4 marzo 2002 e redige un verbale sotto forma d'atto ufficiale entro l'11 marzo 2002. Questo verbale deve essere consegnato senza indugio al Segretariato generale della FMH.

d) Le schede di voto devono essere eliminate al più presto entro 3 mesi e al più tardi entro 6 mesi dalla pubblicazione dei risultati.

e) Il notaio, signora Franziska Iseli ha il diritto di farsi aiutare per questo lavoro da persone che devono essere controllate e istruite a dovere. Per il conteggio dei voti, queste persone devono essere indipendenti dalla FMH e sono obbligate, come il notaio, a mantenere il silenzio.

\footnotetext{
Il cpv. 4 e 5 dell'art. 33 degli Statuti concerne il cambiamento degli Statuti e lo scioglimento della FMH.
} 


\section{Accertamenti in merito allo svolgimento della votazione generale}

Nel corso della votazione generale, il notaio ha fatto i seguenti accertamenti:

\section{Avviso}

La votazione generale è stata annunciata nel «Bollettino dei medici svizzeri" No. 1/2 del 9 gennaio 2002 e No. 5 del 30 gennaio 2002.

\section{Spedizione}

Il materiale per il voto è stato spedito ai membri della FMH, il 4 febbraio 2002. La busta per la votazione conteneva:

a) Una scheda di voto con le tre domande in tedesco, francese e italiano.

b) Le spiegazioni per la votazione generale in tedesco, francese e italiano.

c) Il commento del Comitato centrale della FMH in tedesco, francese e italiano.

d) Un CD-Rom con la documentazione per la votazione, nelle spiegazioni si rendeva attenti al fatto che era possibile ricevere la documentazione anche per iscritto.

e) In base al Regolamento d'esecuzione della FMH, la busta di spedizione vale come documento di legittimazione e al tempo stesso, può essere usata per rimandare la scheda di voto.

Con ciò è possibile costatare che la spedizione è stata svolta secondo il Regolamento d'esecuzione della FMH.

\section{Termini}

a) La votazione generale ha luogo in seguito alla decisione della Camera medica. Il termine secondo la lettera B. / I. / 2. del Regolamento d'esecuzione della $\mathrm{FMH}$, non è rilevante.

b) Il termine di quattro mesi dalla decisione della Camera medica del 13 dicembre 2001, per lo svolgimento della votazione generale è stato rispettato.

c) Il termine di almeno 3 settimane tra la spedizione e la consegna del materiale per la votazione è pure stato rispettato.

Con ciò è possibile costatare che tutti i termini secondo il Regolamento d'esecuzione della FMH sono stati rispettati.

\section{Collaboratori}

Oltre al notaio, signora Franziska Iseli, hanno partecipato allo spoglio delle schede unicamente collaboratori, che sono od erano impiegati nello studio notarile Iseli, di Berna. Tutte queste persone sono completamente indipendenti dalla FMH e come tutto il personale del notariato hanno l'obbligo di mantenere il silenzio, obbligo al quale sono stati resi attenti esplicitamente.
Il notaio, signora Franziska Iseli, costata che tutte le condizioni necessarie per validare lo svolgimento della votazione generale sono adempite.

\section{Risultato della votazione generale}

La votazione generale svolta in base alle sopraccitate norme, ha dato il seguente risultato, in forma anonima:

Domanda 1: Accettate la versione 1.1. della TARMED come struttura tariffaria d'introduzione per le tariffe cantonali LAMal, con la riserva dell'applicazione del piano di ristrutturazione (progetto RE II) entro il 30 giugno 2003 e con la riserva dell'adozione di un regolamento comune in relazione al trasferimento dei dati tra medici e assicuratori?

Schede di voto spedite: 29125

Schede di voto rientrate nel termine del 4 marzo 2002: 15613

delle quali nulle

- per astensione: 835

- altri motivi: 622

schede di voto valide: 14156

Maggioranza assoluta: 7079 voti per il sì

Hanno votato sì: 8949

Hanno votato no: 5207

La maggioranza assoluta per il sì è stata raggiunta e la domanda 1 della votazione generale è stata accettata.

Domanda 2: Accettate la convenzione quadro TARMED del 21 novembre 2001 nell'ambito della LAMal, con la riserva dell'adozione di un regolamento comune in relazione al trasferimento dei dati tra medici e assicuratori?

Schede di voto spedite: 29125

Schede di voto rientrate nel termine del 4 marzo 2002: 15613

delle quali nulle

- per astensione: 946

- altri motivi: 622

schede di voto valide: 14045

Maggioranza assoluta: 7024 voti per il sì

Hanno votato sì: 8343

Hanno votato no: 5702

La maggioranza assoluta per il sì è stata raggiunta e la domanda 2 della votazione generale è stata accettata. 
Domanda 3: Accettate la convenzione tariffaria AINF/AM/AI con un valore iniziale del punto tariffario di Fr. 1.-?

Schede di voto spedite: 29125

Schede di voto rientrate nel termine del 4 marzo 2002: 15613

delle quali nulle

- per astensione: 937

- altri motivi: 622

schede di voto valide: 14054

Maggioranza assoluta: 7028 voti per il sì

Hanno votato sì: 9554

Hanno votato no: 4500

La maggioranza assoluta per il sì è stata raggiunta e la domanda 3 della votazione generale è stata accettata.

Le schede di voto saranno eliminate a partire dal 30 giugno 2002.

\section{Disposizioni finali}

1. Assieme a questo atto ufficiale vengono conservati:
a) allegato No. 1: verbale della Camera medica;
b) allegato No. 2: una scheda di voto con le tre domande di scrutinio;
c) allegato No. 3: le spiegazioni per la votazione generale,
d) allegato No. 4: il commento del Comitato cen- trale;
e) allegato No. 5: un CD-Rom con la documen- tazione della votazione;
f) allegato No. 6: una busta di spedizione vuota.

2. Questo atto ufficiale è redatto in un solo esemplare per la FMH.

Certificato a Berna, nello studio del notaio, signora Franziska Iseli, il sei marzo duemiladue.

D.d. 6 marzo 2002

Il notaio:

sig. signora Franziska Iseli, notaio 